\title{
Dinámica de la fertilización Nitrogenada en el cultivo de brócoli (Brassica Oleracea Var. Híbrido avenger)
}

\section{Dynamics of nitrogen fertilization in the cultivation of Brócoli (brassica oleracea. Hybrid avenger)}

Yamil Cartagena ${ }^{12}$ yamil@colpos.mx

Arturo Galvis ${ }^{2}$ Víctor Volke ${ }^{2}$ Teresa Hernández ${ }^{3}$ Agustín Rodríguez ${ }^{4}$ Rubén Bugarín

${ }^{\mathbf{1}}$ Instituto Nacional Autónomo de Investigaciones Agropecuarias (INIAP-Ecuador). Estación Experimental Santa Catalina. Departamento de Manejo de Suelos y Aguas.

${ }^{2}$ Colegio de Postgraduados (CP-México), Instituto de Recursos Naturales. ${ }^{3}$ Universidad Autónoma Chapingo (UACH-México), Departamento de Irrigación.

${ }^{4}$ Colegio de Mexicano de Especialistas en Recursos Naturales.

${ }^{5}$ Universidad Autónoma de Nayarit.

Artículo recibido en junio de 2016, arbitrado en julio de 2016 y publicado en enero de 2017

RESUMEN

El presente estudio tuvo como finalidad generar a partir de la acumulación de la biomasa vegetativa y comercial una dosis de fertilización nitrogenada utilizando el método racional o balance de nutrientes. Entre las metodologías disponibles para el citado propósito es la del "Método racional o Balance de nutrientes", permitiendo estimar la dosis de fertilización requerida por los cultivos considerando la dinámica nutrimental en el sistema suelo, planta y clima. Se realizó en el Campo Experimental Tlapeaxco, Departamento de Irrigación, Universidad Autónoma Chapingo. Los resultados obtenidos de las variables evaluadas en cada muestreo se sometieron a un análisis de varianza para evaluar el efecto de las láminas de riego $\mathrm{y}$ nitrógeno aplicado, se realizaron pruebas de significancia DMS para láminas de riego y Tukey para el nitrógeno aplicado y sus interacciones a un $\alpha=0.05$ para establecer diferencias entre medias.

Palabras clave: brócoli; fertilización nitrogenada; hibrido
ABSTRACT

The purpose of this study was to generate, from the accumulation of vegetative and commercial biomass, a dose of nitrogen fertilization using the rational method or nutrient balance. Among the available methodologies for the aforementioned purpose is the "Rational Method or Nutrient Balance", allowing to estimate the dose of fertilization required by crops considering the nutritional dynamics in the soil, plant and climate system. It was carried out in the Tlapeaxco Experimental Field, Irrigation Department, Autonomous University of Chapingo. The results obtained from the variables evaluated in each sample were subjected to an analysis of variance to evaluate the effect of the irrigation sheets and applied nitrogen, DMS tests were carried out for irrigation sheets and Tukey for the applied nitrogen and their interactions to $\alpha=0.05$ to establish differences between means.

Key words: broccoli; nitrogen fertilization; hybrid 


\section{INTRODUCCIÓN}

Las proyecciones del crecimiento de la población mundial se incrementarán de 6 mil millones en 1999 a 7 mil millones para el 2020; siendo los países en desarrollo los que tienen las más altas tasas de crecimiento, como consecuencia estas personas deberán tener vivienda, vestido y sobre todo alimento. En los países en desarrollo, la mayoría de los agricultores activos del sector de producción de alimentos son de pequeña escala que forman parte de la pobreza rural. La introducción de nuevos sistemas agrícolas y de tecnologías mejoradas es muy importante para ellos, debido a que la mejora en la productividad resulta no sólo en más alimentos sino también en más ingreso. Entre los principales factores tecnológicos que se encuentran involucrados en el incremento del rendimiento alcanzado en las últimas décadas, se encuentra el uso intensivo de los fertilizantes. Sin embargo su empleo indiscriminado pude ocasionar como resultado un aumento en el contenido de nutrientes cuyo nivel en el suelo sea suficiente y agravar la deficiencia de otros cuyo nivel sea más bajo. Este desequilibrio no favorece la obtención de altos rendimientos ni asegura la rentabilidad de la inversión de los fertilizantes e inclusive se pueden presentar problemas en la salud humana y animal (FAOSTAT, 2001; FAOSTAT, 2009).

La generación de las recomendaciones de fertilización se pueden dividir en tres grupos: sistemas basados principalmente en el análisis químicos de suelos, análisis químicos de plantas y modelos de simulación (Zagal et al, 2003). Las primeras recomendaciones, basadas en el contenido de nitrógeno mineral del suelo al inicio del crecimiento de la planta, surgieron en la década de los setenta; a partir de entonces, diversas ecuaciones para determinar dosis de nitrógeno han sido desarrolladas (Geypens y Vandendriessche, 1996).

Entre las metodologías disponibles para el citado propósito es la del "Método racional o Balance de nutrientes", permitiendo estimar la dosis de fertilización requerida por los cultivos considerando la dinámica nutrimental en el sistema suelo, planta y clima. Sus bases indican que para alcanzar un rendimiento en cierta condición agroecológica, se debe satisfacer un balance entre la demanda del nutrimento y el suministro que se realiza al suelo. Parte, la metodología de la base de que la necesidad de fertilización de un cultivo está dada por la demanda del nutrimento que necesita, la cantidad de nutrimento que suministra el suelo y la eficiencia del fertilizante aplicado (Etchevers et al., 1991; Galvis, 1990; Rodríguez, 1990), según el modelo: "Dosis = (Demanda - Suministro) / Eficiencia". Cuando la demanda de un nutrimento es mayor que el suministro en el suelo, se producirá un déficit que es necesario suplir con la fertilización para el cultivo; en el caso contrario de que la demanda sea menor que el suministro, se aplicará una dosis para mantener la fertilidad del suelo y el rendimiento de un cultivo, con base en criterios agronómicos y experiencia regional (Rodríguez, 1990).

La ventaja de esta metodología es que requiere menor experimentación de campo, por ende es menos costosa y no utiliza análisis de suelo para el cálculo de fertilización nitrogenada, porque el suministro de nitrógeno del suelo se deduce a partir del conocimiento del aporte de nitrógeno de los residuos de cosecha y la fertilización aplicada en el cultivo anterior; llegando a los resultados en corto plazo; lo que lo hace interesante e importante ante la escasez de recursos humanos, económicos y de tiempo. De esta forma el objetivo principal de la metodología es establecer una estrategia de manejo integral agronómico de la fertilización que permita incrementar o en su caso mantener el estado nutrimental del suelo en forma económica para una nutrición rentable de los cultivos sin afectar la sustentabilidad del sistema (Beltrán et al., 1996; Rodríguez et. al., 2001).

En un sistema de agricultura sustentable, la aplicación de dosis adecuadas de nitrógeno está orientada a conseguir un alto retorno económico a través de un rendimiento óptimo y de calidad, pero también es importante minimizar los riesgos de contaminación de aguas superficiales o profundas por lixiviación de nitratos que ocasionan problemas en la salud humana (metahemoglobinemia o síndrome del bebé azul y cáncer gástrico) y de la atmósfera, con gases derivados de procesos como desnitrificación y volatilización (Zagal et al, 2003).

Alfa, Revista de Investigación en Ciencias Agronómicas y Veterinarias Vol. 1, JNo. 1, Enero-A6ril-2017 
El objetivo de esta investigación fue generar a partir de la acumulación de la biomasa vegetativa y comercial una dosis de fertilización nitrogenada utilizando el método racional o balance de nutrientes.

\section{MATERIALES Y MÉTODOS}

La investigación se realizó en el Campo Experimental Tlapeaxco, Departamento de Irrigación, Universidad Autónoma Chapingo. Ubicada en las coordenadas geográficas $19^{\circ}$ $28^{\prime} 58^{\prime \prime}$ de latitud norte y $98^{\circ} 53^{\prime} 27^{\prime \prime}$ de longitud oeste y a 2250 m.s.n.m.

El clima es C(wo)(w)b, que corresponde a la categoría de templado subhúmedo con lluvias en verano, precipitación anual de 637 $\mathrm{mm}$, temperatura anual de $16^{\circ} \mathrm{C}$, evaporación media anual de $1400 \mathrm{~mm}$ y humedad relativa media anual ligeramente superior al $60 \%$ (García, 2004).

El suelo corresponde a un Entisol y sus características físicas y químicas fueron: clase textural arcillosa, capacidad de campo $33.2 \%$, punto de marchitez permanente $24.3 \%$, densidad aparente $1.1 \mathrm{~g}$ cc-3, pH 7.4, conductividad eléctrica $0.5 \mathrm{dSm}-1$, materia orgánica $2.4 \%$, nitrógeno $18.4 \mathrm{mg} \mathrm{kg}-1$, fósforo $50 \mathrm{mg} \mathrm{kg-1} \mathrm{y} \mathrm{potasio} 964 \mathrm{mg} \mathrm{kg}-1$.

El trasplante se realizó el 3 de octubre del 2013, utilizando el material biológico de brócoli, híbrido Avenger (Sakata 2009).

El diseño experimental fue de parcela dividida en bloques completamente al azar, con 8 tratamientos, 4 repeticiones y 32 unidades experimentales. En la Parcela Grande se colocaron dos láminas de riego (3.0 y $6.0 \mathrm{~mm}$ ) y en la Sub Parcela se aplicaron dosis crecientes de nitrógeno $(80,160,240$ y $320 \mathrm{~kg}$ ha-1). El sistema de riego utilizado fue por goteo. La aplicación del fertilizante químico se realizó manualmente, fraccionando el nitrógeno (Urea) en 3 aplicaciones $(1,30$ y 50 días después del trasplante) y el fósforo (Fosfato diamónico) al momento del trasplante (50 kg ha-1). La unidad experimental para cada tratamiento fue de $8.4 \mathrm{~m}$ de largo por $1.8 \mathrm{~m}$ de ancho, con una distancia entres surcos de $1.20 \mathrm{~m}$ y entre plantas de $0.3 \mathrm{~m}$, obteniéndose una densidad de 22000 plantas ha- 1 .

Las variables evaluadas fueron: peso fresco, peso seco, nitrógeno, nitrato y nitrito acumulados; en la biomasa vegetativa (tallos y hojas) con 5 muestreos $(20,40,60,80$ y 100 días después del trasplante) y en la biomasa comercial (inflorescencia) con 5 muestreos $(80,85,90,95$ y 100 días después del trasplante).

Los resultados obtenidos de las variables evaluadas en cada muestreo se sometieron a un análisis de varianza para evaluar el efecto de las láminas de riego y nitrógeno aplicado, se realizaron pruebas de significancia DMS para láminas de riego y Tukey para el nitrógeno aplicado y sus interacciones a un $\alpha$ $=0.05$ para establecer diferencias entre medias. Los análisis estadísticos se realizaron en el paquete estadístico Statistical Analysis Sistem 9.0 (SAS, 1999).

El peso fresco de la biomasa vegetal y comercial se tomó de una planta completa en el surco central de la parcela neta; en el laboratorio se realizaron dos lavados, uno con agua corriente y otro con agua destilada, se secaron al ambiente y pesaron en una balanza de precisión (Ohaus Adventurer Pro) y los resultados se expresaron en g planta-1.Para obtener el peso seco a los materiales vegetales obtenidos, se secaron en un horno de ventilación forzada (Shel Labcom) a una temperatura de $65^{\circ} \mathrm{C}$ hasta que se obtuvo un peso constante, se pesó en una balanza de precisión (Ohaus Adventurer Pro) y los resultados se expresaron en g planta-1.

El nitrógeno acumulado, se determinó analíticamente la concentración de Nitrógeno por el método de Semimicro-Kjeldahl (Alcantar y Sandoval, 1999); este valor obtenido se multiplicó con el peso seco de la biomasa, dividió entre 100 y los resultados se expresaron en mg planta- 1 .

El nitrato acumulado, se determinó analíticamente la concentración de Nitrato por el método de Cataldo (1975); se calculó dividiendo la concentración de nitrato para 10000 , se multiplicó con el peso seco de la biomasa, dividió entre 100 y multiplicó por 1000, expresando los resultados en miligramos por planta.

El nitrito acumulado, se determinó analíticamente la concentración de Nitrito por el método de Griess Ilosvay (Bremner, 1965); este valor obtenido se calculó dividiendo la concentración de nitrito para 10000, se multiplicó con el peso seco de la biomasa, dividió entre 100 y se multiplicó por 1000000 , 
expresando los resultados en microgramos por planta.

\section{RESULTADOS Y DISCUSIÓN}

\section{Peso Fresco}

El análisis de la varianza para fresco de la biomasa vegetativa en el factor láminas de riego detectó diferencias estadísticas a los 20 y 40 días después del trasplante y no mostró diferencias estadísticas en los demás muestreos; para el factor dosis de nitrógeno aplicado y la interacción, no se detectaron diferencias estadísticas en los muestreos realizados (Tabla 1). Para el peso fresco de la biomasa comercial, en las láminas de riego detectó diferencias estadísticas sólo en el muestreo a los 95 días después del trasplante y para la dosis de nitrógeno y la interacción no se presentaron diferencias estadísticas (ver tabla 2).

El peso fresco de la biomasa comercial a los 100 días después del trasplante se le denominó rendimiento con el cual se aplicó una función cuadrática; observándose que para la lámina de riego de $3.0 \mathrm{~mm}$, el máximo valor fue de $922.0 \mathrm{~g}$ planta-1 y para la lámina de riego de $6.0 \mathrm{~mm}$, con $1079.3 \mathrm{~g}$ planta- 1 , con las dosis de $240 \mathrm{~kg}$ ha-1, siendo alto el rendimiento con lo reportado por Castellanos (1998). El efecto del incremento en rendimiento con el de la humedad del suelo fue de $5 \%$, siendo muy bajo para la cantidad de agua aplicada.

\section{Peso seco}

El análisis de la varianza del peso seco de la biomasa vegetativa en el factor láminas de riego presentó diferencia estadística a los 20 días después del trasplante y no hubo diferencias estadísticas para los demás factores en estudio y muestreos realizados (Tabla 1). El peso seco de la biomasa comercial mostró diferencias estadísticas a los 85 días después del trasplante y no existieron diferencias estadísticas para las dosis de nitrógeno aplicado y la interacción (Tabla 2).

\section{Nitrógeno acumulado}

Se evaluó el nitrato acumulado en la biomasa comercial, desde los 80 hasta los 100 días después del trasplante, cuya tendencia ajustó una función logística normal, representándose como una curva típica sigmoidal. La fase inicial presentó un incremento lineal muy lento que duró hasta los 85 días después del trasplante, acumulando menos del 20\%. En la siguiente fase se apreció una tendencia exponencial que duró desde los 85 a los 95 días después del trasplante, en el cual se notó un desarrollo muy marcado de la inflorescencia. La fase final ocurrió entre los 95 y 100 días después del trasplante, en donde disminuye su crecimiento e inicia el proceso de floración en el cual baja su calidad comercial (ver tabla 3 ).

\section{Nitrato acumulado}

El nitrato acumulado en la biomasa comercial, se aplicó una función cuadrática inversa en la que se presentaron una fase inicial con incremento exponencial positivo hasta los 90 días después del trasplante, con un valor máximo de $65 \mathrm{mg}$ planta- 1 con la lámina de riego de $3 \mathrm{~mm}$ y la siguiente fase mostró un incremento exponencial negativo (ver tabla 3).

\section{Nitrito acumulado}

El nitrito acumulado tuvo una función cuadrática inversa en la que el valor máximo correspondió a 4 g30 planta-1 con la lámina de riego de $3 \mathrm{~mm}$ (Tabla 3 ).

La correlación entre nitrito acumulado y nitrato acumulado mostró un valor de 0.7 indicando que existe una buena asociación entre variables (Figura 1).

Se realizó una representación gráfica de la información del rendimiento, nitrógeno acumulado en la planta y la dosis de nitrógeno aplicado, en tres cuadrantes; en los que el cuadrante a) establece la relación entre la dosis de nitrógeno aplicado (eje horizontal izquierdo) y rendimiento (eje vertical superior), el cuadrante b) describe la variación entre el Nitrógeno acumulado (eje horizontal derecho) y el rendimiento (eje vertical superior) y en el cuadrante c) muestra la variación entre el Nitrógeno acumulado en la planta (eje horizontal derecho) y el Nitrógeno aplicado (eje vertical inferior). Las variaciones en las relaciones han sido descritas por Van-Keulen (1981) para arroz, 
Loomis y Connor (1992), Rouanet (1994) para maíz y trigo y Berti et al. (2000) en quinua.

En la figura 2 se apreció muy marcadamente el efecto de la lámina de riego de $6 \mathrm{~mm}$ en el rendimiento de brócoli y también se presentó una relación directa de este efecto en la cantidad de nitrógeno acumulado en la planta. Por lo tanto la eficiencia en el uso del Nitrógeno disminuye conforme se incrementa la dosis de nitrógeno aplicado, cuando este alcanza un determinado valor, un incremento de fertilizante no se traduce en incremento de rendimiento, y en algunos casos puede provocar la caída de los mismos; además la cantidad de Nitrógeno en el suelo será mayor, lo cual provoca el riesgo de contaminación. En la relación entre el Nitrógeno acumulado en la planta y el Nitrógeno aplicado, correspondió a una función lineal en la cual la pendiente nos indica la eficiencia de la fertilización nitrogenada (44\%) y la ordenada en el origen es el suministro de nitrógeno en el suelo (148 $\mathrm{kg}$ ha-1). Con estos valores obtenidos se aplicó el modelo simplificado propuesto por Rodríguez (1990) para calcular la dosis de Nitrógeno estimado, obteniéndose valores muy cercanos a la dosis de nitrógeno aplicado (ver tabla 4).

Tabla 1. Cuadrados medios y niveles de significancia para peso fresco y peso seco en la biomasa vegetativa en brócoli.

\begin{tabular}{|c|c|c|c|c|c|c|c|c|c|c|c|}
\hline \multirow[t]{2}{*}{ F.V } & \multirow[t]{2}{*}{ G.L. } & \multirow{2}{*}{\multicolumn{2}{|c|}{20}} & \multirow[b]{2}{*}{40} & \multicolumn{4}{|c|}{ Días después del trasplante } & & \multirow{2}{*}{\multicolumn{2}{|c|}{100}} \\
\hline & & & & & \multicolumn{3}{|c|}{60} & \multicolumn{2}{|l|}{80} & & \\
\hline & & \multicolumn{10}{|c|}{$\begin{array}{l}\text { Peso } \\
\text { fresco }\end{array}$} \\
\hline Bloques & 3 & 216 & NS & 22222 & NS & 119832 & NS & 1182996 & NS & 1914608 & \\
\hline Láminas & 1 & 131.2 & * & 472.3 & * & 11037.2 & NS & 63287.0 & NS & 1058414 & No \\
\hline Error (a) & 3 & 7.1 & & 973.7 & & 18914.4 & & 93833.7 & & 392447.0 & \\
\hline Nitrógeno & 3 & 48.4 & NS & 11627.2 & NS & 26037.0 & NS & 83789.7 & NS & 35045.4 & NS \\
\hline$L \times N$ & 3 & 34.2 & NS & 1553.8 & NS & 40736.7 & NS & 155136.3 & NS & 30534.3 & NS \\
\hline Error (b) & 18 & 31.1 & & 1339.1 & & 25290.3 & & 148406.5 & & 107147.4 & \\
\hline \multirow[t]{2}{*}{ C. V. (\%) } & & 32.1 & & 25.9 & & 20.2 & & 26.2 & & 18.2 & \\
\hline & & \multicolumn{10}{|c|}{ Peso seco } \\
\hline Total & 31 & & & & & & & & & & \\
\hline Bloques & 3 & 0.3 & NS & 20.3 & NS & 198.2 & NS & 2290.2 & NS & 2154.8 & NS \\
\hline Láminas & 1 & 1.7 & * & 0.5 & NS & 76.1 & NS & 842.5 & NS & 555.2 & NS \\
\hline Error (a) & 3 & 0.1 & & 12.0 & & 107.3 & & 932.5 & & 3984.0 & \\
\hline Nitrógeno & 3 & 0.8 & NS & 134.2 & ** & 342.1 & NS & 496.1 & NS & 1324.8 & NS \\
\hline L x N & 3 & 0.5 & NS & 15.0 & NS & 309.1 & NS & 2372.4 & NS & 565.6 & NS \\
\hline Error (b) & 18 & 0.5 & & 14.7 & & 136.4 & & 1537.8 & & 1695.8 & \\
\hline C. V. (\%) & & 30.3 & & 23.9 & & 13.2 & & 24.3 & & 19.9 & \\
\hline
\end{tabular}

F.V. = Fuente de variación, G.L. $=$ Grados de libertad, NS = No significativa, ${ }^{*}=$ Significativa $\alpha(=$ $0.05) \mathrm{y}$

** = Altamente significativa $(\alpha=0.01)$. 
Tabla 2. Cuadrados medios y niveles de significancia para peso fresco y peso seco en la biomasa comercial en brócoli.

\begin{tabular}{|c|c|c|c|c|c|c|c|c|c|c|c|}
\hline \multirow[t]{2}{*}{ F.V } & \multirow[t]{2}{*}{ G.L. } & \multicolumn{10}{|c|}{ Días después del trasplante } \\
\hline & & \multicolumn{2}{|l|}{80} & \multicolumn{2}{|l|}{85} & \multicolumn{2}{|l|}{90} & \multicolumn{2}{|l|}{95} & \multicolumn{2}{|l|}{100} \\
\hline & & \multicolumn{10}{|c|}{$\begin{array}{l}\text { Peso } \\
\text { fresco }\end{array}$} \\
\hline Bloques & 3 & 6199.8 & * & 6511.0 & NS & 4711.9 & NS & 6220.7 & NS & 27523.0 & NS \\
\hline Láminas & 1 & 2078.0 & NS & $\begin{array}{r}73409 . \\
6 \\
\end{array}$ & NS & $\begin{array}{r}104128 . \\
8\end{array}$ & NS & $\begin{array}{r}150906 . \\
4\end{array}$ & * & $\begin{array}{r}141642 . \\
3\end{array}$ & NS \\
\hline Error (a) & 3 & 667.5 & & 9250.0 & & 17727.5 & & 9968.7 & & $\begin{array}{r}149344 . \\
8\end{array}$ & \\
\hline Nitrógeno & 3 & 2395.1 & NS & $\begin{array}{r}12900 . \\
4\end{array}$ & NS & 8659.6 & NS & 38063.3 & NS & 12767.1 & NS \\
\hline L X N & 3 & 1531.8 & NS & 7569.0 & NS & 2849.2 & NS & 2102.9 & NS & 2427.7 & NS \\
\hline Error (b) & 18 & 2551.7 & & 9839.2 & & 13410.2 & & 14479.0 & & 17213.8 & \\
\hline \multirow[t]{2}{*}{ C. V. (\%) } & & 62.1 & & 23.3 & & 20.8 & & 15.7 & & 13.6 & \\
\hline & & \multicolumn{10}{|c|}{ Peso seco } \\
\hline Total & 31 & & & & & & & & & & \\
\hline Bloques & 3 & 63.3 & * & 94.0 & NS & 56.7 & NS & 8.6 & NS & 224.2 & NS \\
\hline Láminas & 1 & 20.6 & NS & 419.1 & * & 541.9 & NS & 685.3 & NS & 43.5 & NS \\
\hline Error (a) & 3 & 5.8 & & 28.9 & & 193.5 & & 83.3 & & 884.2 & \\
\hline Nitrógeno & 3 & 23.0 & NS & 74.2 & NS & 20.5 & NS & 92.3 & NS & 35.3 & NS \\
\hline$L \times N$ & 3 & 18.1 & NS & 51.9 & NS & 37.3 & NS & 52.6 & NS & 19.9 & NS \\
\hline Error (b) & 18 & 26.4 & & 80.2 & & 86.3 & & 75.8 & & 120.9 & \\
\hline C. V. (\%) & & 57.3 & & 22.0 & & 17.0 & & 13.9 & & 13.9 & \\
\hline
\end{tabular}

F.V. $=$ Fuente de variación, G.L. $=$ Grados de libertad, NS $=$ No significativa, ${ }^{*}=$ Significativa $\alpha(=$ $0.05) \mathrm{y}^{* *}=$ Altamente significativa $(\alpha=0.01)$.

Tabla 3. Acumulación de nitrógeno, nitrato y nitrito en la biomasa comercial de brócoli, con dos láminas de riego y cuatro dosis de nitrógeno aplicado.

\begin{tabular}{|c|c|c|c|}
\hline \multicolumn{2}{|c|}{ Combinación } & \multirow[t]{2}{*}{ Ecuación } & \multirow{2}{*}{$\begin{array}{l}\text { Cuadrado } \\
\text { Medio }\end{array}$} \\
\hline Láminas & $\mathbf{N}_{\text {apl }}$ & & \\
\hline \multirow{3}{*}{ L3.0 } & & Nitrógeno & \\
\hline & N80 & $\mathrm{N}_{\mathrm{T}}=2991.5\left[1+20.7 \times \mathrm{e}^{(-0.1753 \times \mathrm{Ddt})}\right]^{-1}$ & 71590.1 \\
\hline & N160 & $\mathrm{N}_{\mathrm{T}}=3523.0\left[1+15.7 \times \mathrm{e}^{(-0.1526 \mathrm{xdt})}\right]^{-1}$ & 101772.0 \\
\hline \multirow{5}{*}{ L6.0 } & N240 & $\mathrm{N}_{\mathrm{T}}=1.2 \times 10^{11}\left[1+3.5 \times 10^{8} \times \mathrm{e}^{(-0.088 \times \mathrm{Ddt})}\right]^{-1}$ & 248339.0 \\
\hline & N320 & $\mathrm{N}_{\mathrm{T}}=3207.3\left[1+40.1 \times \mathrm{e}^{(-0.2381 \times \mathrm{Ddt})}\right]^{-1}$ & 85926.5 \\
\hline & N80 & $\mathrm{N}_{\mathrm{T}}=2774.3\left[1+947.5 \times \mathrm{e} \mathrm{e}^{(-0.5151 \times \mathrm{Ddt})}\right]^{-1}$ & 81231.1 \\
\hline & N160 & $\mathrm{N}_{\mathrm{T}}=3691.0\left[1+27.0 \times \mathrm{x} \mathrm{e}^{(-0.1980 \mathrm{xdd})}\right]^{-1}$ & 96345.8 \\
\hline & N240 & $\mathrm{N}_{\mathrm{T}}=2910.6[1+1929.7 \times \mathrm{x} \text { (-0.6122 x Ddt) }]^{-1}$ & 10486926.0 \\
\hline & N320 & $\mathrm{N}_{\mathrm{T}}=3302.6\left[1+86.4 \times \mathrm{xe}^{(-0.3267 \mathrm{x} \mathrm{Ddt})}\right]^{-1}$ & 58716.4 \\
\hline \multicolumn{4}{|c|}{ Nitrato } \\
\hline \multirow[t]{4}{*}{ L3.0 } & N80 & $\mathrm{NO}_{3}=\left(0.0009 \mathrm{D}_{\mathrm{dt}^{2}}{ }^{2}-0.0408 \mathrm{D}_{\mathrm{dt}}+0.4682\right)^{-1}$ & 60.5 \\
\hline & N160 & $\mathrm{NO}_{3}=\left(0.0013 \mathrm{D}_{\mathrm{dt}^{2}}{ }^{2}-0.0567 \mathrm{D}_{\mathrm{dt}}+0.6158\right)^{-1}$ & 89.6 \\
\hline & N240 & $\mathrm{NO}_{3}=\left(0.0003 \mathrm{D}_{\mathrm{dt}^{2}}{ }^{-0.0175} \mathrm{D}_{\mathrm{dt}}+0.2368\right)^{-1}$ & 66.1 \\
\hline & N320 & $\mathrm{NO}_{3}=\left(0.0004 \mathrm{D}_{\mathrm{dt}^{2}}{ }^{-0.0217} \mathrm{D}_{\mathrm{dt}}+0.3014\right)^{-1}$ & 55.2 \\
\hline L6.0 & N80 & $\mathrm{NO}_{3}=\left(0.0008 \mathrm{D}_{\mathrm{dt}^{2}}{ }^{2}-0.0361 \mathrm{D}_{\mathrm{dt}}+0.4092\right)^{-1}$ & 72.2 \\
\hline
\end{tabular}

Alfa, Revista de Investigación en Ciencias Agronómicas y Veterinarias Vol. 1, Nro. 1, Enero-Abri-2017 


\begin{tabular}{|c|c|c|c|}
\hline \multicolumn{2}{|c|}{ Combinación } & \multirow[t]{2}{*}{ Ecuación } & \multirow{2}{*}{$\begin{array}{l}\text { Cuadrado } \\
\text { Medio }\end{array}$} \\
\hline Láminas & Napl & & \\
\hline & N160 & $\mathrm{NO}_{3}=\left(0.0005 \mathrm{D}_{\mathrm{dt}^{2}}-0.0245 \mathrm{D}_{\mathrm{dt}}+0.3048\right)^{-1}$ & 56.8 \\
\hline & N240 & $\mathrm{NO}_{3}=\left(0.0004 \mathrm{D}_{\mathrm{dt}^{2}}{ }^{-}-0.0198 \mathrm{D}_{\mathrm{dt}}+0.2523\right)^{-1}$ & 100.8 \\
\hline & N320 & $\mathrm{NO}_{3}=\left(0.0003 \mathrm{D}_{\mathrm{dt}^{2}}{ }^{2}-0.0187 \mathrm{D}_{\mathrm{dt}}+0.2688\right)^{-1}$ & 30.5 \\
\hline \multicolumn{4}{|c|}{ Nitrito } \\
\hline L3.0 & N80 & $\mathrm{NO}_{2}=\left(0.001 \mathrm{D}_{\mathrm{dt}^{2}}-0.06 \mathrm{D}_{\mathrm{dt}}+0.7\right)^{-1}$ & 3.0 \\
\hline & N160 & $\mathrm{NO}_{2}=\left(0.001 \mathrm{D}_{\mathrm{dt}^{2}}-0.05 \mathrm{D}_{\mathrm{dt}}+0.6\right)^{-1}$ & 4.6 \\
\hline & N240 & $\mathrm{NO}_{2}=\left(0.0009 \mathrm{D}_{\mathrm{dt}^{2}}{ }^{2}-0.04 \mathrm{D}_{\mathrm{dt}}+0.5\right)^{-1}$ & 2.2 \\
\hline & N320 & $\mathrm{NO}_{2}=\left(0.0005 \mathrm{D}_{\mathrm{dt}^{2}}{ }^{2}-0.02 \mathrm{D}_{\mathrm{dt}}+0.3\right)^{-1}$ & 32.6 \\
\hline \multirow[t]{4}{*}{ L6.0 } & N80 & $\mathrm{NO}_{2}=\left(0.0001 \mathrm{D}_{\mathrm{dt}^{2}}{ }^{2}-0.01 \mathrm{D}_{\mathrm{dt}}+0.1\right)^{-1}$ & 89.0 \\
\hline & N160 & $\mathrm{NO}_{2}=\left(0.0008 \mathrm{D}_{\mathrm{dt}^{2}}-0.04 \mathrm{D}_{\mathrm{dt}}+0.5\right)^{-1}$ & 6.3 \\
\hline & N240 & $\mathrm{NO}_{2}=\left(0.0004 \mathrm{D}_{\mathrm{dt}^{2}}^{2}-0.02 \mathrm{D}_{\mathrm{dt}}+0.3\right)^{-1}$ & 22.5 \\
\hline & N320 & $\mathrm{NO}_{2}=\left(0.0003 \mathrm{D}_{\mathrm{dt}^{2}}{ }^{2}-0.01 \mathrm{D}_{\mathrm{dt}}+0.2\right)^{-1}$ & 47.5 \\
\hline
\end{tabular}

Ddt= Días después del trasplante

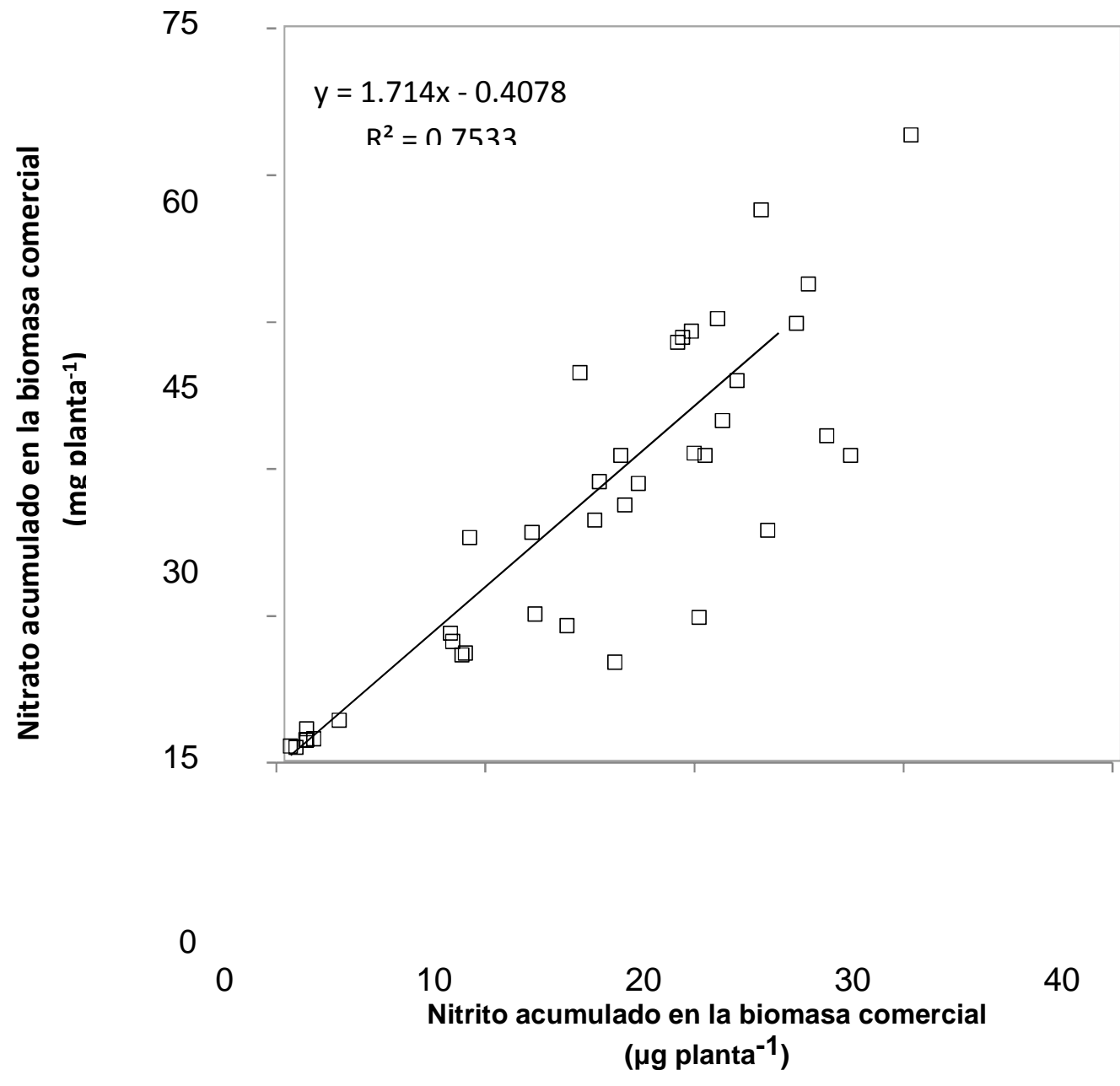

Figura 1. Relación el Nitrito acumulado ( $\mu$ g planta $^{-1}$ ) y Nitrato acumulado (mg planta-1) en la biomasa comercial del brócoli. 


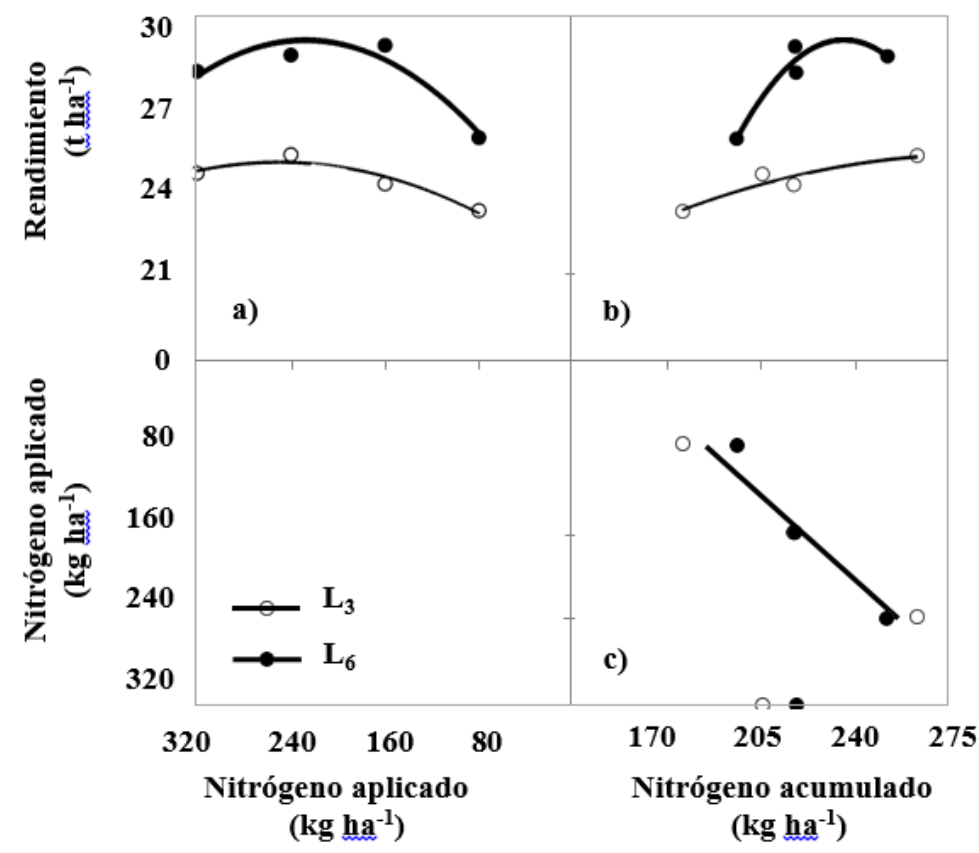

$80 \quad 160 \quad 240 \quad 320$

Figura 2. Relación a) entre dosis de Nitrógeno aplicado (kg ha-1) y rendimiento ( $\left.\left.\mathrm{t} \mathrm{ha}{ }^{-1}\right) ; \mathrm{b}\right)$ entre Nitrógeno acumulado en la planta $\left(\mathrm{kg} \mathrm{ha}^{-1}\right)$ y rendimiento $\left(\mathrm{t} \mathrm{ha}^{-1}\right)$ y c) entre dosis de Nitrógeno aplicado ( $\left.\mathrm{kg} \mathrm{ha}^{-1}\right)$ y Nitrógeno acumulado en la planta $\left(\mathrm{kg} \mathrm{ha}^{-1}\right)$, para dos láminas de riego $(3.0 \mathrm{y}$ $6.0 \mathrm{~mm})$.

Tabla 4. Rendimiento, demanda de nitrógeno y dosis de nitrógeno (aplicado y estimado), con suministro en el suelo de $148 \mathrm{~kg} \mathrm{ha}^{-1}$ y eficiencia de aplicación del fertilizante del $44 \%$.

\begin{tabular}{|c|c|c|c|c|c|}
\hline \multicolumn{2}{|c|}{ Combinación } & \multirow{3}{*}{$\begin{array}{c}\text { Rendimient } \\
\text { o } \\
\text { t ha }^{-1}\end{array}$} & \multirow{3}{*}{$\begin{array}{l}\text { Demanda } \\
\text { nitrógeno }\end{array}$} & \multicolumn{2}{|c|}{ Dosis de nitrógeno } \\
\hline Láminas & Dosis de & & & Aplicado & Estimado \\
\hline de riego & nitrógeno & & & kg ha-1 $^{-1}$ & \\
\hline \multirow[t]{5}{*}{$\mathrm{L}_{3}$} & N80 & 23.2 & 175.7 & 80 & 63 \\
\hline & N16 & 24.1 & 217.0 & 160 & 156 \\
\hline & $\mathrm{N}_{24}$ & 25.1 & 262.8 & 240 & 260 \\
\hline & 0 & & & & \\
\hline & $\begin{array}{c}\mathrm{N} 32 \\
0\end{array}$ & 24.5 & 205.4 & 320 & - \\
\hline \multirow[t]{4}{*}{$\mathrm{L}_{6}$} & $\begin{array}{c}\text { N80 } \\
0\end{array}$ & 25.7 & 195.7 & 80 & 108 \\
\hline & $\begin{array}{c}\mathrm{N}_{16} \\
0\end{array}$ & 28.9 & 217.5 & 160 & 157 \\
\hline & $\begin{array}{c}\mathrm{N}_{24} \\
0\end{array}$ & 28.6 & 251.8 & 240 & 235 \\
\hline & $\begin{array}{c}\text { N32 } \\
0\end{array}$ & 28.0 & 217.8 & 320 & - \\
\hline
\end{tabular}


CONCLUSIONES

El rendimiento máximo de brócoli se obtuvo con la dosis de $240 \mathrm{~kg}$ ha- 1 con 20 y 23 t ha-1 para la lámina de riego de 3.0 y $6.0 \mathrm{~mm}$ respectivamente; siendo altos estos rendimientos en comparación con la producción nacional que se encuentra en promedio de $14 \mathrm{t}$ ha-1.

Se obtuvieron los valores de los parámetros del método racional para el cultivo de brócoli, híbrido Avenger, como son: demanda de $10 \mathrm{~kg}$ ha-1 por tonelada de inflorescencia producida, suministro del suelo de $148 \mathrm{~kg}$ ha-1 y eficiencia de la aplicación del fertilizante del 44\%.La aplicación de dosis y niveles crecientes de nitrógeno y láminas de riego, respectivamente, no tuvieron un efecto significativo en el incremento del rendimiento que fue alrededor del $10 \%$, debido a que el suministro inicial de nitrógeno fue alto ocasionando una baja eficiencia del fertilizante nitrogenado y afectando también a la economía y ambiente.

La cantidad de nitrato y nitrito acumulados fue mayor a los 90 días después del trasplante, pudiendo ocasionar problemas en la salud, cuando se ingieren altas dosis de brócoli en la dieta diaria; debiéndose mencionar que la mejor época de cosecha debe ser antes o después de esta fecha mencionada, aunque la calidad en lo referente a compactación, color y madurez de las flores en la inflorescencia.

REFERENCIAS

Alcantar, G. y Sandoval M. (1999). Manual de análisis químico vegetal. Publicación especial 10

Sociedad mexicana de la Ciencia del Suelo. Chapingo, México

Beltrán, R., Volke H. y Núñez R. (1996). Un modelo de balance nutrimental para generar recomendaciones de fertilización para arroz en suelos de Cuba. Serie Cuadernos de Edafología 26. Montecillos, México

Berti, M. Wilckens, R. Hevia, F. Serri, H. Vidal, I. y Méndez, C. (2000). Fertilización nitrogenada en Quínoa (Chenopodium quinua WILLD). Ciencia e Investigación Agraria 27 (2): 81-90. Chile
Bremner, J. M. (1965). Inorganic nitrogen. In: C .C. Black et al. (Eds) Methods of soil analysis. PART II. Agronomy N9: 684-687 Am. Soc. of Agron. Madison. USA

Castellanos, R., J. Z. (1998). El seguimiento de la nutrición del brócoli en los sistemas de fertirrigación Plantaciones modernas. AGROSEM año 3, 1: 137-152. México, México. DF.

Cataldo, D.A. (1975). Rapid colorimetric determination of nitrate plant tissue by nitration of acid salicilic. Commun. Soil Sci. and plant analysis. 6:71-80. USA.

Etchevers, B., J. Rodríguez S., J. y A. Galvis S. (1991). Generación de recomendaciones de fertilización mediante un enfoque sistemático racional. Terra 9(1):3-10.

Galvis S., A. (1990). Validación de las normas de fertilización para maíz generadas con un modelo simplificado, con las obtenidas en la experimentación de campo. Tesis de Maestro en Ciencias. Colegio de Postgraduados. Montecillos, México.

García E. (2004). Modificaciones al sistema de clasificación climática de Koppen. Quinta edición. Universidad Autónoma de México, México. DF.

Geypens, M., and H. Vandendriessche. (1996). Advisory systems for nitrogen fertilizer recommendations. Plant Soil 181:31-38.

FAOSTAT. (2001). Statistical databases. http://apps.fao.org.

FAO STAT. (2009). Agriculture Statistics. http://faostat.fao.org/site/567/DesktopDefau lt.aspx?PageID=567\#ancor

Loomis, R. y Connors, D. (1992). Crop ecology: productivity and management in agricultural systems. Cambridge University Press. Melksham, England. 538 p.

Rodríguez, S. J. (1990). La fertilización de los cultivos: un método racional. $1^{\underline{a}}$ edición. Facultad de Agronomía, Pontificia Universidad Católica de Chile, Santiago, Chile.

Rodríguez, J.; Pinochet; D., y Matus F. (2001). Fertilización de los cultivos. SantiagoTalca-Valdivia. I.S.B.N.: 956-288-880-0.

Rouanet, J. (1994). Eficiencia fisiológica de uso de nitrógeno por cultivos anuales en futura agricultura sustentable. Agricultura técnica 54(2): 169-179. Chile. 
Sakata, (2009). Sakata seed México. Catálogo de hortalizas. http://www.sakata.com/ paginas/avenger.htm.

http://www.sakata.com/uploads/catalog_ pdfs/vegetable/vegcatalog_spanish.pdf.

Van-Keulen, H. (1981). Modelling the interaction of water and nitrogen. In: Montheith, J. and C. Webb (Ed). Soil water and nitrogen in mediterranean environment. Developments in plant and soil sciences. Vol. Nijhauff and Junk Publishers. The Hague. P.: 205-229

Zagal, E. Hirzel, J. y Vidal, I. (2003). Evaluación de la recomendación de fertilización nitrogenada para cultivos anuales en suelos de Origen volcánico usando un modelo de simulación. Agricultura Técnica. vol.63, n.1. pp. 94-104. Chile 